A N N A L E S Annales de Bretagne et des Pays de l'Ouest

\title{
La Cornouaille politique 1870-1914. Étude sur le berceau
} de la Bretagne républicaine

Jean Le Bihan

\section{(2) OpenEdition}

Édition électronique

URL : http://journals.openedition.org/abpo/3681

DOI : $10.4000 /$ abpo.3681

ISBN : 978-2-7535-6497-8

ISSN : 2108-6443

Éditeur

Presses universitaires de Rennes

Édition imprimée

Date de publication : 30 juin 2017

Pagination : 178-180

ISBN : 978-2-7535-6495-4

ISSN : 0399-0826

\section{Référence électronique}

Jean Le Bihan, «La Cornouaille politique 1870-1974. Étude sur le berceau de la Bretagne républicaine»,

Annales de Bretagne et des Pays de l'Ouest [En ligne], 124-2 | 2017, mis en ligne le 17 juillet 2017, consulté le 23 septembre 2020. URL : http://journals.openedition.org/abpo/3681 ; DOI : https:// doi.org/10.4000/abpo.3681 
que l'on savait ou devinait déjà par ailleurs, ce travail de S. Jahan présente l'intérêt de montrer une fois encore la pertinence des échelles d'observation locale et provinciale, la finesse des stratifications sociales et le lent maillage relationnel qui permet l'engloutissement de l'individu dans le collectif. L'étranger est aussi un miroir dans lequel se révèle et se reflète le fonctionnement social, à l'échelle d'un village, d'une province ou de la France.

Philippe JARNOUX

LuCAS, Maurice, La Cornouaille politique 1870-1914. Étude sur le berceau de la Bretagne républicaine, Paris, Les Indes savantes, 2014, 541 p.

Cet ouvrage constitue la version publiée d'une thèse de doctorat dirigée par Yves Le Gallo et soutenue il y a déjà plus de 30 ans, en 1983 précisément, devant l'université de Bretagne occidentale. Comme son titre l'indique, l'objectif de la recherche dont les résultats sont ici synthétisés visait à examiner comment la Troisième République avait été accueillie en Cornouaille, cette partie méridionale du département du Finistère dont Maurice Lucas fixe la limite nord à une ligne courant approximativement de Roscanvel à Brennilis. Le territoire étudié correspond en fait à l'aire couverte par quatre circonscriptions législatives (la première de Châteaulin, les deux de Quimper, celle de Quimperlé) puis cinq à partir de 1910 quand la seconde circonscription de Quimper est divisée en deux, savoir la circonscription de Douarnenez d'un côté et celle de Pont-l'Abbé de l'autre. Ce choix n'a rien d'anodin. Il s'explique par l'importance cruciale accordée aux élections législatives, que l'auteur a érigées en observatoire privilégié pour décrire et analyser le développement du courant républicain en Cornouaille. Construit en conséquence, le corpus documentaire se compose pour l'essentiel de rapports préfectoraux et de police conservés dans la série $M$ des archives départementales du Finistère, ainsi que de journaux locaux. Au bout du compte, l'évolution qui se fait jour à la lecture de ce méticuleux travail peut se dire en une formule : la républicanisation dans la modération. Reprenons le cours de ce petit demi-siècle d'histoire politique en distinguant pour ce faire trois périodes.

Au début des années 1870, le département du Finistère reporte rapidement au profit de la République le ferme soutien qu'il avait auparavant apporté à l'Empire. En font foi les victoires républicaines remportées lors des élections municipales et législatives partielles de 1871, suivies, deux ans plus tard, par celle, très nette, du modéré Gustave Swiney, que l'auteur interprète comme l'expression d'un rejet de l'Ordre moral. Ainsi, dès cette époque, la Cornouaille, et particulièrement ses côtes, se montrent autrement accueillantes à la République que le nord du département, mais une république toute d'ordre et de sagesse, comme en témoigne encore le fait que la cause communarde ne suscite aucun élan de sympathie localement.

Du milieu des années 1870 au tournant du xxe siècle, les républicains modérés règnent pour ainsi dire sans partage sur la représentation parlementaire cornouaillaise, si ce n'est pendant la quatrième législature, le remplacement du scrutin d'arrondissement par le scrutin de liste départemental entraînant la déroute complète des républicains finistériens en 1885 - mais une déroute vouée à rester unique du fait même du retour au scrutin d'arrondissement quatre ans plus tard. Émergent alors de grandes figures du département tels Louis Hémon, député de la première circonscription de Quimper de 1876 à 1885 puis de 1889 à 1912, ou bien encore James de Kerjégu, qui domine sans concurrence aucune la circonscription 
de Quimperlé entre 1889 et 1908. Entre tous ces notables, de multiples nuances existent qui donnent à voir la grande variété interne d'une culture républicaine en continuelle réélaboration : ainsi Hémon, anticlérical, hostile au socialisme, soucieux de l'éducation du peuple, est-il un représentant caractéristique de la Gauche républicaine, quand Kerjégu, lui, nourrit une conception à la fois éclairée et conservatrice de l'ordre social et se montre farouchement opposé à la guerre religieuse. L'affirmation de ces républicains modérés marginalise peu à peu les grands propriétaires monarchistes. Sur l'autre bord, elle bâillonne aussi les tenants du radicalisme, ainsi qu'en témoigne l'impact somme toute limité de l'épisode boulangiste en Cornouaille. Du moins à l'occasion des élections législatives car, localement, la gauche radicale marque des points à la fin du siècle, que ce soit dans le pays bigouden, à Douarnenez ou dans le Cap-Sizun. Il est toutefois difficile de se faire une idée précise de la manière dont la République conquiert les localités cornouaillaises. On devine que ce sont les petites villes qui s'y rallient les premières; mais il faut tenir compte des rapports de force politiques locaux, déterminants, qui expliquent, par exemple, que Douarnenez, terre républicaine s'il en est, passe à droite en 1896. Compte tenu de la documentation dont il dispose, $M$. Lucas ne peut guère que nous proposer des éclairages partiels à cet égard.

Les premières années du $\mathrm{xx}^{\mathrm{e}}$ siècle voient la situation évoluer sur fond de recomposition du jeu politique national et de dégradation des conditions de vie des populations côtières cornouaillaises à la suite de la crise de la pêche sardinière. Le fait le plus notable est alors la montée en puissance du courant radical, dont la première percée parlementaire est l'œuvre de Georges Le Bail, élu député de la seconde circonscription de Quimper en 1902. Le Bail, déjà maire de Plozévet et conseiller général, est "l'incarnation locale du Bloc " (p. 284). Très hostile au collectivisme, il entend d'abord œuvrer à l'amélioration des conditions de vie du petit peuple cornouaillais, celui des cultivateurs et des pêcheurs, et il sait, par ailleurs, faire montre d'une prudence de bon aloi en matière religieuse. Cette victoire ne reste pas isolée : huit ans plus tard, la Cornouaille compte trois députés radicaux sur cinq, dont le propre fils de Le Bail. Mais l'élan se tasse alors : à la veille de la guerre, les deux Le Bail, père et fils, ne sont réélus que sur le fil et la circonscription de Quimperlé est reconquise par les modérés. Deux ordres d'explications peuvent être avancés pour rendre compte de cet essoufflement. Joue d'abord la concurrence des socialistes. Leur audience a en effet beaucoup augmenté sur les côtes; ils ont même ravi la mairie de Concarneau en 1911. Reste que, si actifs soient-ils dans certaines localités, ils ne peuvent encore compter que sur des "victoires d'enclaves" (p. 498). Il faut donc aussi souligner la grande force de résistance des modérés, emblématiquement représentés, à la veille de la Grande Guerre, par le nouveau député de Quimper Maurice Bouilloux-Lafont.

On insistera, en définitive, sur l'originalité de la voie empruntée par la Cornouaille : en se ralliant aussi vite, aussi facilement à la République, elle se distingue avec force du Léon voisin où, tout au contraire, les idées républicaines peinent tant à se faire accepter; mais la Cornouaille n'est pas pour autant le simple reflet de l'évolution nationale en terre bretonne, car les poussées à gauche y sont systématiquement atténuées ou différées, qu'il s'agisse de la montée en force des radicaux dans les années 1880 ou de celle des socialistes dans les années 1900. Cette originalité politique, M. Lucas ne prétend pas en rénover l'explication. Il préfère se placer sous le patronage du Tableau politique. La Cornouaille, c'est, disait Siegfried, la terre d'élection du " catholicisme bleu ». Il faut entendre par là qu'elle se caractérise par une certaine indépendance à l'égard des autorités traditionnelles - à l'égard du clergé et, du fait de la rareté des grands domaines, à l'égard de la noblesse - mais aussi que les Cornouaillais demeurent attachés à la religion catholique en tant que 
telle. L'esprit d'indépendance qui anime la société cornouaillaise explique, toujours selon Siegfried, la vitesse de son ralliement à la République et autorise bien à faire de cette partie du Finistère le " berceau de la Bretagne républicaine ", tandis que sa piété explique son opposition à l'anticléricalisme d'État et donc, in fine, sa propension au modérantisme. Il y aurait là un " tempérament politique ", notion dont l'auteur revendique l'usage et défend la pertinence (p. 66), que la conjoncture des années 1870-1914 n'aurait en somme que peu altéré. On peut en effet penser qu'un certain dynamisme économique (qualification prudente vu l'absence d'étude économique de synthèse sur la Bretagne du temps) a contribué à renforcer l'adhésion générale à la République, quand bien même, sur les côtes, la crise du début du $\mathrm{Xx}^{\mathrm{e}}$ siècle a plutôt poussé à son dépérissement.

Très classiquement conçu, l'ouvrage de $\mathrm{M}$. Lucas n'intègre certes pas les nouveaux axes de réflexion dont l'histoire politique s'est enrichie au cours de ces dernières décennies, au contact de la science politique notamment. Ainsi ne s'interroget-il pas sur le processus de politisation de la société cornouaillaise, ne réfléchit-il pas vraiment aux gestes, rituels et technologies du suffrage, passe-t-il très vite sur la sociologie des députés, les fondements et les formes de leur autorité. On aurait toutefois mauvaise grâce à reprocher à l'auteur de ne pas avoir pris en compte ces questionnements qui, pour l'essentiel, se sont imposés postérieurement à la soutenance de sa thèse. Deux autres regrets peuvent plus légitimement saisir le lecteur. Le premier est de pure méthode : le livre manque cruellement de documents d'appui propres à faciliter la lecture, savoir des cartes et des tableaux récapitulant les résultats des scrutins que l'auteur examine si minutieusement. Le second intéresse le fond. On aurait beaucoup aimé que l'auteur entre en discussion avec la thèse de Patrick Pierre (Les Bretons et la République. La construction de l'identité bretonne sous la Troisième République, Rennes, PUR, 2001), elle aussi inscrite dans le sillage de Siegfried et dont le regard sur l'évolution politique de la Cornouaille, s'il ne diverge pas fondamentalement de celui de M. Lucas, ne s'y superpose pas tout à fait. Sur la question territoriale, en particulier, un débat fécond aurait pu s'engager. Que penser, par exemple, du fait que P. Pierre distingue pour sa part le "modèle politique " du pays de Châteaulin - cette région sise entre Basse-Cornouaille et Léon dont, soit dit en passant, Siegfried ne savait que penser lui-même - de celui de la Cornouaille maritime? Comment, par ailleurs, articuler l'intéressante typologie des cantons cornouaillais élaborée par P. Pierre en fonction de l'époque de leur ralliement à la République (dès les années 1870, lors du Ralliement, suite à la Séparation), avec l'analyse développée par M. Lucas dans son livre, moins fine spatialement mais beaucoup plus précise eu égard à l'histoire politique propre de la Cornouaille? Ce n'étaient là que deux objets de discussion possibles, parmi d'autres.

Ces remarques n'enlèvent évidemment rien de son intérêt à ce livre fort bien écrit qui retrace minutieusement une étape clé de l'histoire cornouaillaise, finistérienne et bretonne, et qui, par surcroît, fournit un utile éclairage sur les campagnes électorales, leur violence et leurs débordements, ainsi que sur l'infinie variété que présente l'idéologie républicaine dès lors que celle-ci est observée au " ras d'un terroir". 